\title{
Study of Some Cosmological Parameters for Interacting New Holographic Dark Energy Model in $f(T)$ Gravity
}

\author{
Chayan Ranjit] \\ Department of Mathematics, Egra S. S. B. College, Purba Medinipur-721429, W.B. India.
}

\author{
Prabir Rudra 2 \\ Department of Mathematics, Asutosh College, Kolkata-700 026, India.
}

\begin{abstract}
The present work is based on the idea of an interacting framework of new holographic dark energy with cold dark matter in the background of $f(T)$ gravity. Here, we have considered the flat modified Friedmann universe for $f(T)$ gravity which is filled with new Holographic dark energy and dark matter. We have derived some cosmological parameters like Deceleration parameter, EoS parameter, State-finder parameters, Cosmographic parameters, $\mathrm{Om}$ parameter and graphically investigated the nature of these parameters for the above mentioned interacting scenario. The results are found to be consistent with the accelerating universe. Also we have graphically investigated the trajectories in $\omega-\omega^{\prime}$ plane for different values of the interacting parameter and explored the freezing region and thawing region in $\omega-\omega^{\prime}$ plane. Finally, we have analyzed the stability of this model.
\end{abstract}

\section{Introduction}

Accelerated phenomenon of the universe is now well established observable fact but the physical origin of this acceleration is unknown till now. There are so many observational studies like the different luminosity type Ia supernovae (SNIa) 1, 2, 3, 4, in associated with Large scale Structure 5, 6] and Cosmic Microwave Background (CMB) 7, 8, 9, 10, 11 radiation which strongly support that our universe is currently expanding with an acceleration. Many candidates are considered as the main responsible for this observational facts of expanding scenario which triggered out a new type of matter which violates the strong energy condition i.e., $\rho+p<0$. The energy density of such mysterious matters which have negative pressure thats leads to cosmic acceleration, are known as Dark Energy (DE) [12, 13, 14, 15, 16. Recent observational studies indicate that Dark energy occupies about $73 \%$ of the energy of our universe, while dark matter contribution is $\sim 23 \%$ and the usual baryonic matter is $\sim 4 \%$. Dark energies are separated depending on their equation of state (EOS). In the case of quintessence type DE, the EOS is $-1<\omega<1 / 3$, whereas for phantom type DE, EOS is $\omega<-1$ but in the case of quintom type DE, the EOS can cross the phantom divide $\omega=-1$ from both sides. The most charming and simplest candidate for DE is the cosmological constant $\Lambda$. However, it is known to all that the cosmological constant suffers two serious theoretical problems, i.e. the cosmological constant problem and the coincidence problem. Hence, for seeking the most reliable model for describing the DE phenomenon, different methods have been adopted and for that different dynamical DE models have been developed.

There are another point of view to describe that acceleration scenario. Recently many researchers consider Modified Gravity as the alternating candidate of dark energy in support of accelerating phenomenon. The basic idea of Modified Gravity is that a concept of modification of gravitational theory and as a result it provides a very natural gravitational alternative for exotic matter. Without commencing negative kinetic term of dark energies, Modify Gravity models can explain the phantom or non-phantom or quintom phase of the universe, not only that, it also illustrates about the early time inflation then transition from deceleration to late time acceleration. The most popular String/M-theory also supports Modify Gravity models. In recent day scenario, it is treated as the rival of General Relativity. Although there are some rigid constraints in modify gravity theory, some particular form of modified gravity obtains a great attention of current researchers. Ricci curvature $R$ of Lagrangian is one of them. Different form of Ricci curvature have been considered 17, 18, 19, 20, 21 to explain the accelerating phase of the Universe. In this regards, some remarkable work was done by Nojiri et al [22, 23] where they considered the modified Lagrangian as $R+R^{m}+R^{-m}$ and as a result they obtained an inflation at an early stage and also a late time of accelerated expansion. These result lead to open new window of gravity dependent modern research. The most reliable modified gravity models incorporate $f(R)$ gravity (where $R$ represent the Ricci Scalar Curvature) [24, 25, 26, $f(T)$ gravity (where $T$ is the torsion scalar) $27,28,29, f(G)$ gravity

\footnotetext{
${ }^{1}$ chayanranjit@gmail.com

2 prudra.math@gmail.com
} 
(where $G$ represents the Gauss-Bonnet invariant) [30, 31, $f(R, T)$ gravity [32, 33, $f(R, G)$ gravity [34, 35] and so on. Recently, reconstruction between different dark energy models become a very appealing scenario in modern cosmological study. In 2008, Setare et al. 36] studied the cosmological proposition of the correspondence of Holographic dark energy model and Gauss-Bonnet dark energy model. That showed the way to explanation of the accelerated expansion of the universe by imposing specific constraints. In [37. Liu et al. talked about the New Agegraphic Dark Energy (NADE) model in the framework of the Brans-Dicke theory with the help of EoS and deceleration parameters and finally they showed that the EoS parameter has a quintom-like behavior for that model and it indicates the accelerated expansion of the universe. The NADE in $f(R)$ gravity model has been discussed in [38] and as a result it found that there may exist a phantom-like universe. Also in 39] the NADE model is correspond with Horava-Lifshitz gravity which specified that the accelerated expanding universe is consistent with cosmological observations. The reconstruction of Entropy-Corrected Holographic Dark Energy (ECHDE) in the $f(G)$ gravity also been investigated to explain the expanding universe with acceleration in [40. Recently the HDE model in the framework of the $f(G)$ gravity has been discussed in [41] and the different phenomenon for the accelerating universe are explained.

The main purpose of this work is to analyzed some cosmological parameters for interacting new Holographic dark energy model in $f(T)$ gravity model. For this reason, homogeneous, isotropic modified FRW for $f(T)$ gravity has been considered and after that conservation equations for interacting new Holographic dark energy and dark matter have been solved in Section 2. Then density and pressure are converted into dimensionless quantity. After that Deceleration parameter, EoS parameter, $\omega-\omega^{\prime}$ plane analysis, State-finder parameters, Cosmographic parameters and Om parameter are converted in terms of current density, pressure and Hubble expansion rate parameter and graphically analyzed in Section 3 respectively. In Section 4, stability of this model have been analyzed. Finally, the results of the paper are summarized in Section 5.

\section{Field equations and their solutions}

The metric of a spatially flat homogeneous and isotropic universe in FRW model is given by

$$
d s^{2}=d t^{2}-a^{2}(t)\left[d r^{2}+r^{2}\left(d \theta^{2}+\sin ^{2} \theta d \phi^{2}\right)\right]
$$

where $a(t)$ is the scale factor.

The Einstein field equation are given by

$$
H^{2}=\frac{1}{3} \rho
$$

and

$$
2 \dot{H}+3 H^{2}=-p
$$

where $\rho$ and $p$ are energy density and isotropic pressure respectively (choosing $8 \pi G=c=1$ )and $H=\frac{\dot{a}}{a}$ is the Hubble parameter.

Let us we start with $f(T)$ gravity models where the action is given by

$$
S=\frac{1}{2 \kappa^{2}} \int d x^{4}\left[\sqrt{-g} f(T)+L_{m}\right]
$$

where $T$ is the torsion scalar, $f(T)$ is general differentiable function of the torsion and $L_{m}$ corresponds to the matter Lagrangian, $\kappa^{2}=8 \pi G=1$. Therefore the field equations for modified FRW model are given by

$$
3 H^{2}=\rho_{M G}=\rho+\rho_{T}
$$

and

where

$$
2 \dot{H}+3 H^{2}=-p_{M G}=-\left(p+p_{T}\right)
$$

$$
\rho_{T}=\frac{1}{2}\left(2 T f_{T}-f+6 H^{2}\right), \quad p_{T}=-\frac{1}{2}\left[-8 \dot{H} T f_{T T}+(2 T-4 \dot{H}) f_{T}-f+4 \dot{H}+6 H^{2}\right]
$$

and $T=-6 H^{2}$.

We consider the power law model $f(T)=T^{n}$, where $n$ is a constant. Restricting ourselves to $n=2$ for simplicity, we obtain

$$
\rho_{T}=3 H^{2}\left(18 H^{2}+1\right), \quad p_{T}=-2 \dot{H}-3 H^{2}\left(24 \dot{H}+18 H^{2}+1\right)
$$

Also we consider that our universe is filled with new Holographic dark energy and dark matter. So we assume, $\rho=\rho_{x}+\rho_{m}$ and $p=p_{x}+p_{m}$. Here $\rho_{x}, p_{x}$ and $\rho_{m}, p_{m}$ are respectively the energy density and pressure for new holographic dark energy and dark matter. Although the dark matter has negligible pressure, i.e., $p_{m} \approx 0$ but here we taken into account the non-zero value of $p_{m}$ (which is very small value).

The energy conservation equation is given by

$$
\dot{\rho}+3 \frac{\dot{a}}{a}(\rho+p)=0
$$


Now we consider the model of interaction between dark matter and the new holographic dark energy model, through a phenomenological interaction term Q. Keeping into consideration the fact that the Supernovae and CMB data determine that decay rate should be proportional to the present value of the Hubble parameter. The interaction term describes the energy flow between the two fluids. Therefore the conservation equation (9) becomes

$$
\dot{\rho}_{x}+3 H\left(\rho_{x}+p_{x}\right)=-Q
$$

and

$$
\dot{\rho}_{m}+3 H\left(\rho_{m}+p_{m}\right)=Q
$$

There are many different forms of $\mathrm{Q}$ in the literature, we choose the following form of $\mathrm{Q}$ as

$$
Q=3 \delta H \rho_{m}
$$

where $\delta$ (may positive or negative)is the interaction parameter. In our present study we take $\delta \geq 0$.

The new Holographic dark energy density is given by

$$
\rho_{x}=3\left(\alpha H^{2}+\beta \dot{H}\right)
$$

where $\alpha$ and $\beta$ are constants. Now the EOS of dark matter is given by $p_{m}=\omega_{m} \rho_{m}$, where $\omega_{m}$ is very small and so the equation (11) becomes

$$
\rho_{m}=\rho_{m 0} a^{-3\left(1+\omega_{m}-\delta\right)}
$$

and consequently from the equation (5), we have

$$
H^{2}=\frac{\sqrt{-\frac{\beta}{a^{3\left(1+\omega_{m}\right)}}}\left[\alpha \sqrt{-\frac{a^{3\left(1+\omega_{m}\right)}}{\beta}} J_{-U}(V) C+\sqrt{-\frac{6 a^{3 \delta} \rho_{m 0}}{\beta}}\left(2 J_{-1+U}(V)+\left(J_{-1-U}(V)-J_{1-U}(V)\right) C\right)\right]}{36\left(J_{U}(V)+J_{-U}(V) C\right)}
$$

where $J_{U}(V)$ is a Bessel function of first kind, $U=\frac{2 \alpha}{3 \beta\left(1-\delta+\omega_{m}\right)}$ and $V=-\frac{\sqrt{\frac{32}{3} \rho_{m 0}} a^{-\frac{3}{2}\left(1-\delta+\omega_{m}\right)}}{\beta\left(1-\delta+\omega_{m}\right)}$ and $\rho_{m 0}$ is the present value of matter density (at $a=1$ ) and $C$ is the arbitrary integration constant.

Now we define:

$$
\begin{array}{ll}
\widetilde{H}^{2}=\frac{H^{2}}{H_{0}^{2}} ; & \\
\widetilde{\rho}_{m}=\frac{\rho_{m}}{3 H_{0}^{2}} ; & \widetilde{\rho}_{x}=\frac{\rho_{x}}{3 H_{0}^{2}} ; \quad \widetilde{\rho}_{T}=\frac{\rho_{T}}{3 H_{0}^{2}} ; \\
\widetilde{p}_{m}=\frac{p_{m}}{3 H_{0}^{2}} ; & \widetilde{p}_{x}=\frac{p_{x}}{3 H_{0}^{2}} ; \quad \widetilde{p}_{T}=\frac{p_{T}}{3 H_{0}^{2}} ; \\
\widetilde{\Omega}_{m 0}=\frac{\rho_{m 0}}{3 H_{0}^{2}} ; \quad \widetilde{\Omega}_{x 0}=\frac{\rho_{x 0}}{3 H_{0}^{2}} ; \quad \widetilde{\Omega}_{T 0}=\frac{\rho_{T 0}}{3 H_{0}^{2}} ;
\end{array}
$$

where $H_{0}$ is the present value of the Hubble parameter, $\widetilde{H}$ is the Hubble expansion rate, current density parameters are $\Omega_{m 0}$ and $\Omega_{x 0}$ for matter and dark energy respectively. The scale factor $a$ is expressed in terms of redshift $\mathrm{z}$ as

$$
a=\frac{1}{1+z}
$$

Therefore from the equations (5)-(8), (10)-(16) we have

$$
\begin{gathered}
\widetilde{\rho}_{m}=\widetilde{\Omega}_{m 0}(1+z)^{3\left(1-\delta+\omega_{m}\right)} \\
\widetilde{\rho}_{T}=\left(18 H^{2}+1\right) \widetilde{H}^{2} \\
\widetilde{\rho}_{x}=\alpha \widetilde{H}^{2}-\frac{\beta(1+z)}{2} \frac{d \widetilde{H}^{2}}{d z} \\
\widetilde{p}_{m}=\omega_{m} \widetilde{\Omega}_{m 0}(1+z)^{3\left(1-\delta+\omega_{m}\right)} \\
\widetilde{p}_{T}=\frac{1}{3 \beta}\left[\left((2 \alpha-3 \beta)+18 H^{2}(4 \alpha-3 \beta)\right) \widetilde{H}^{2}-2\left(36 H^{2}+1\right) \widetilde{\rho}_{x}\right]
\end{gathered}
$$

and

$$
\widetilde{p}_{x}=-\delta \widetilde{\rho}_{m}-\alpha \widetilde{H}^{2}+\frac{1}{3}(\alpha+\beta)(1+z) \frac{d \widetilde{H}^{2}}{d z}-\frac{1}{6} \beta(1+z)^{2} \frac{d^{2} \widetilde{H}^{2}}{d z^{2}}
$$

where we use the relations $\frac{\dot{H}}{H^{2}}=-\frac{(1+z)}{2 \widetilde{H}^{2}} \frac{d \widetilde{H}^{2}}{d z} \& \frac{\ddot{H}}{H^{3}}=\frac{(1+z)}{2 \widetilde{H}^{2}} \frac{d \widetilde{H}^{2}}{d z}+\frac{(1+z)^{2}}{2 \widetilde{H}^{2}} \frac{d^{2} \widetilde{H}^{2}}{d z^{2}}$. 


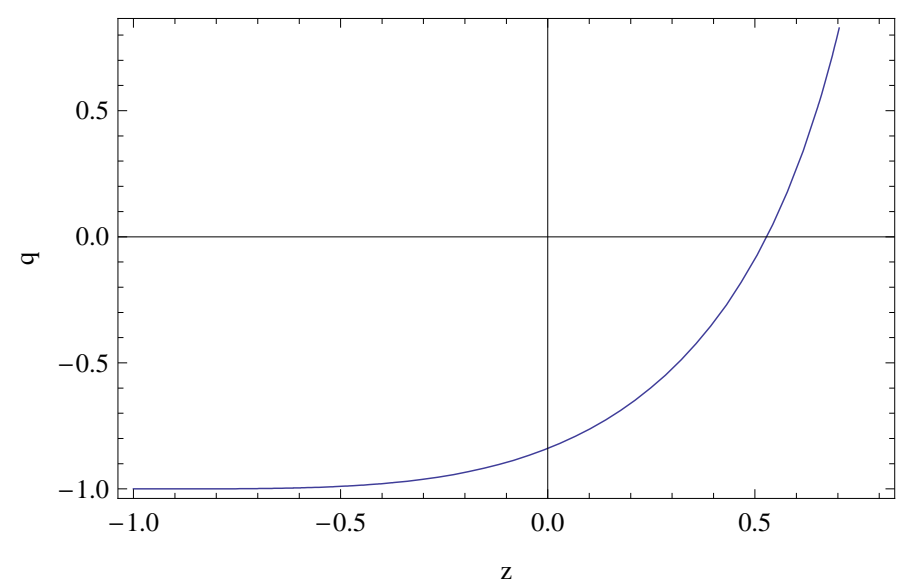

Fig.1

Fig.1 shows the variation of deceleration parameter (q) against the redshift (z).

\section{Some Well-Known Cosmological Parameters}

\subsection{Deceleration Parameter}

Deceleration parameter plays a very important role for any cosmological model to determine the cosmic acceleration. A negative value of q represents cosmic acceleration, whereas a positive q gives a decelerating universe. The deceleration parameter is given by

$$
q=-1-\frac{\dot{H}}{H^{2}}=-1+\frac{(1+z)}{2 \widetilde{H}^{2}} \frac{d \widetilde{H}^{2}}{d z}
$$

and in this present scenario, the expression for $q$ is obtained as

$$
\begin{aligned}
& q=-1+\left(1 8 ( \frac { 1 } { 1 + z } ) ^ { - 1 + \frac { 3 } { 2 } ( 1 + \omega _ { m } ) } ( J _ { U } ( V ) + J _ { - U } ( V ) C ) H _ { 0 } ^ { 2 } \left(\left(( \frac { 1 } { 1 + z } ) ^ { 1 - \frac { 3 } { 2 } ( 1 + \omega _ { m } ) } \sqrt { - \frac { 1 } { \beta } \beta } \left(\left(\frac{1}{1+z}\right)^{\frac{3}{2}\left(1+\omega_{m}\right)} \alpha \sqrt{-\frac{1}{\beta}} J_{-U}(V) C+\right.\right.\right.\right. \\
& \left.\left.\sqrt{6}\left(\frac{1}{1+z}\right)^{3 \delta / 2}\left(2 J_{-1+U}(V)+\left(J_{-1-U}(V)-J_{1-U}(V)\right) C\right) \sqrt{-\frac{\rho_{m 0}}{\beta}}\right)\left(1+\omega_{m}\right)\right) /\left(24\left(J_{U}(V)+J_{-U}(V) C\right) H_{0}^{2}\right)+ \\
& \left(( \frac { 1 } { 1 + z } ) ^ { - \frac { 3 } { 2 } ( 1 + \omega _ { m } ) } \sqrt { - \frac { 1 } { \beta } } \beta \left(-3 \sqrt{\frac{3}{2}}\left(\frac{1}{1+z}\right)^{1+\frac{3 \delta}{2}} \delta\left(2 J_{-1+U}(V)+\left(J_{-1-U}(V)-J_{1-U}(V)\right) C\right) \sqrt{-\frac{\rho_{m 0}}{\beta}}-\right.\right. \\
& \left.\left.\left.\left.\frac{3}{2}\left(\frac{1}{1+z}\right)^{1+\frac{3}{2}\left(1+\omega_{m}\right)} \alpha \sqrt{-\frac{1}{\beta}} J_{-U}(V) C\left(1+\omega_{m}\right)\right)\right) /\left(36\left(J_{U}(V)+J_{-U}(V) C\right) H_{0}^{2}\right)\right)\right) / \\
& \left(\sqrt{-\frac{1}{\beta}} \beta\left(\left(\frac{1}{1+z}\right)^{\frac{3}{2}\left(1+\omega_{m}\right)} \alpha \sqrt{-\frac{1}{\beta}} J_{-U}(V) C+\sqrt{6}\left(\frac{1}{1+z}\right)^{3 \delta / 2}\left(2 J_{-1+U}(V)+\left(J_{-1-U}(V)-J_{1-U}(V)\right) C\right) \sqrt{-\frac{\rho_{m 0}}{\beta}}\right)\right)
\end{aligned}
$$

The deceleration parameter is plotted against the redshift parameter in Fig 1.

\subsection{EoS Parameter}

The nature of matter content of the universe is depicted by the Equation of State (EoS) parameter $\omega$. Basically its gives an idea about the era of the universe. When $\omega=0,1 / 3$ and 1 , it can be predicted that the universe is in the dust era, the radiation era and stiff fluid respectively whereas $\omega=-1 / 3,-1$ and $\omega<-1$ represent the quintessence DE, $\Lambda$ CDM era and Phantom era respectively. The EoS parameter can be obtained as

$$
\omega=\frac{2 q-1}{3}
$$




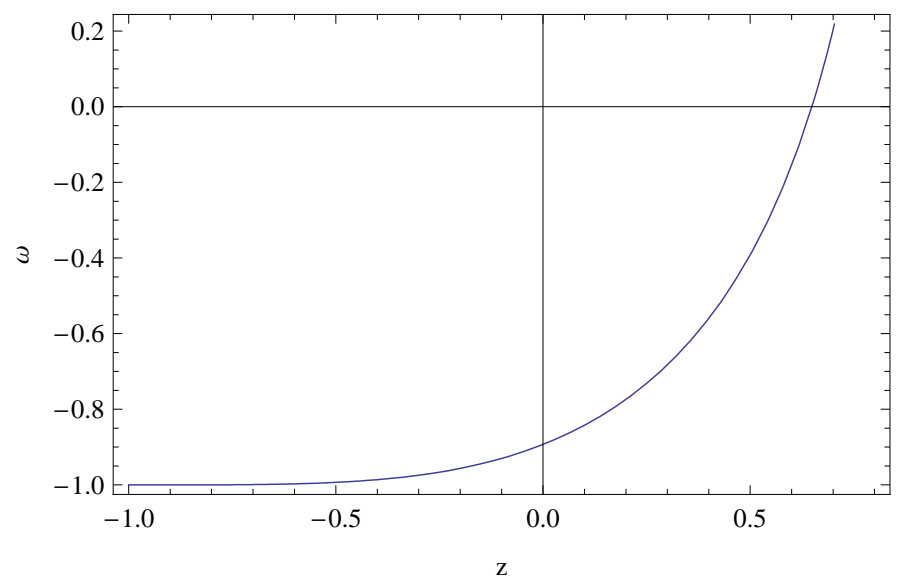

Fig.2

Fig.2 depicts the change of EoS parameter against the redshift.

Here we have obtained it for the given model as:

$$
\begin{aligned}
& \omega=-\frac{1}{3} \\
& +\frac{2}{3}\left[-1+\left(1 8 ( \frac { 1 } { 1 + z } ) ^ { - 1 + \frac { 3 } { 2 } ( 1 + \omega _ { m } ) } ( J _ { U } ( V ) + J _ { - U } ( V ) C ) H _ { 0 } ^ { 2 } \left(\left(( \frac { 1 } { 1 + z } ) ^ { 1 - \frac { 3 } { 2 } ( 1 + \omega _ { m } ) } \sqrt { - \frac { 1 } { \beta } \beta } \left(\left(\frac{1}{1+z}\right)^{\frac{3}{2}\left(1+\omega_{m}\right)} \alpha \sqrt{-\frac{1}{\beta}} J_{-U}(V) C+\right.\right.\right.\right.\right. \\
& \left.\left.\sqrt{6}\left(\frac{1}{1+z}\right)^{3 \delta / 2}\left(2 J_{-1+U}(V)+\left(J_{-1-U}(V)-J_{1-U}(V)\right) C\right) \sqrt{-\frac{\rho_{m} 0}{\beta}}\right)\left(1+\omega_{m}\right)\right) /\left(24\left(J_{U}(V)+J_{-U}(V) C\right) H_{0}^{2}\right)+ \\
& \left(( \frac { 1 } { 1 + z } ) ^ { - \frac { 3 } { 2 } ( 1 + \omega _ { m } ) } \sqrt { - \frac { 1 } { \beta } \beta } \left(-3 \sqrt{\frac{3}{2}}\left(\frac{1}{1+z}\right)^{1+\frac{3 \delta}{2}} \delta\left(2 J_{-1+U}(V)+\left(J_{-1-U}(V)-J_{1-U}(V)\right) C\right) \sqrt{-\frac{\rho_{m 0}}{\beta}}-\right.\right. \\
& \left.\left.\left.\left.\frac{3}{2}\left(\frac{1}{1+z}\right)^{1+\frac{3}{2}\left(1+\omega_{m}\right)} \alpha \sqrt{-\frac{1}{\beta}} J_{-U}(V) C\left(1+\omega_{m}\right)\right)\right) /\left(36\left(J_{U}(V)+J_{-U}(V) C\right) H_{0}^{2}\right)\right)\right) / \\
& \left.\left(\sqrt{-\frac{1}{\beta}} \beta\left(\left(\frac{1}{1+z}\right)^{\frac{3}{2}\left(1+\omega_{m}\right)} \alpha \sqrt{-\frac{1}{\beta}} J_{-U}(V) C+\sqrt{6}\left(\frac{1}{1+z}\right)^{3 \delta / 2}\left(2 J_{-1+U}(V)+\left(J_{-1-U}(V)-J_{1-U}(V)\right) C\right) \sqrt{-\frac{\rho_{m 0}}{\beta}}\right)\right)\right]
\end{aligned}
$$

The plot of the EoS parameter against the redshift parameter is obtained in Fig 2.

\section{$3.3 \quad \omega-\omega^{\prime}$ Plane Analysis}

The $\omega-\omega^{\prime}$ (where prime denotes derivatives with respect to $x=\ln a$ ) plane analysis is very significant tool in our modern days cosmological analysis. Basically, it has been used to distinguish different DE models through trajectories on its plane. At first this approach was proposed by Caldwell and Linder in 2005 [2]. Initially, this method has been applied on quintessence DE model which leads to two classes of its plane, one of which has been known as thawing region where the area occupied by the region as $\omega>0$ for $\omega^{\prime}<0$ on $\omega-\omega^{\prime}$ plane while the other known as freezing region where $\omega<0$ for $\omega^{\prime}<0$ and it is also notable that the expansion of the universe is comparatively more accelerating in freezing region. Recently, this tool has been widely applied to other well-known dynamical DE models such as more general form of quintessence [43, Pilgrim DE [44, 45, 46], quintom [47], phantom [4], polytropic DE [49] and others. Here we try to analyze and emphasize such regions with the help of this method. Now $\omega^{\prime}$ can be obtained by differentiating Eq. (27) with respect to $x$. The plot for $\omega$ versus $\omega^{\prime}$ for our predicted model with different value of $\delta=1,0,0.5$ are given in Figs. 3-5 respectively. Also it can be observed from those figures that the freezing regions are possible for noninteracting scenario i.e., $\delta=0$ and for interacting scenario with $\delta=0.5$, whereas for high interaction scenario, $\delta=1.0$ the thawing region is a possibility with the assumption that the other parameters are fixed at same value as the previous cases. Again it can be observed that $\omega^{\prime} \rightarrow 0$ when $\omega \rightarrow-1$ i.e., $\Lambda$ CDM limit may be possible only the in case when $\delta=0$.

\subsection{Statefinder Parameters}

Statefinder Parameters solved the problem of discriminating between the various candidates for dark energy model. In 2003 Sahni et al [50] proposed the trajectories in the $\{r, s\}$ plane corresponding to different cosmological models to depict qualitatively different behavior. The statefinder diagnostic along with future SNAP observations may perhaps be used to discriminate between different dark energy models. The above statefinder diagnostic pair are defined as follows: 


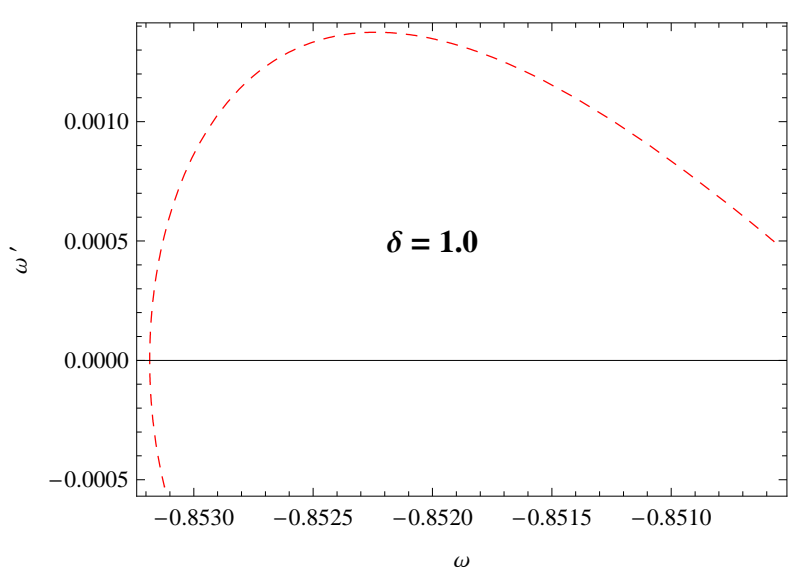

Fig.3

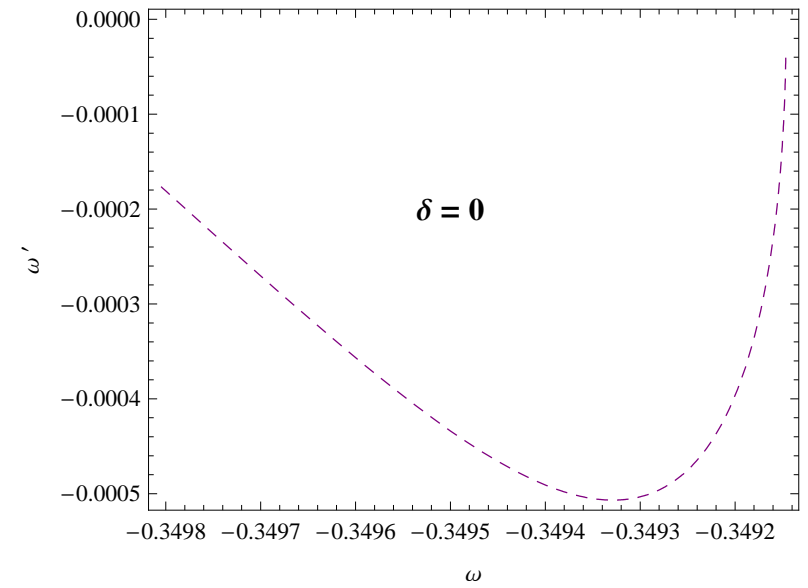

Fig.4

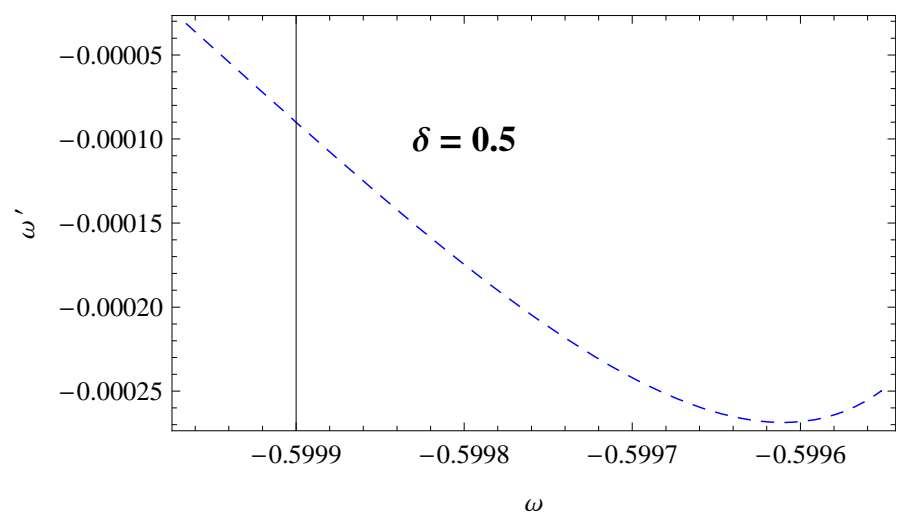

Fig.5

Fig. 3-5 show the plot of $\omega-\omega^{\prime}$ for $\delta=1.0,0,0.5$ respectively with other parameters fixed at $C=0.005$, $\omega_{m}=0.3, \rho_{m 0}=0.01, \alpha=0.95, \beta=-2.1$ and $H_{0}=73$. 


$$
r=1+3 \frac{\dot{H}}{H^{2}}+\frac{\ddot{H}}{H^{3}} \quad \text { and } \quad s=\frac{r-1}{3\left(q-\frac{1}{2}\right)}
$$

where $q$ is the deceleration parameter defined by $q=-1-\frac{\dot{H}}{H^{2}}$ and $H$ is the Hubble parameter. These parameters

Now $\mathrm{r}$ and $\mathrm{s}$ can be written in terms of Hubble parameter $\mathrm{H}$ in the following forms:

$$
r=1+3 \frac{\dot{H}}{H^{2}}+\frac{\ddot{H}}{H^{3}}
$$

and

$$
s=-\frac{3 H \dot{H}+\ddot{H}}{3 H\left(2 \dot{H}+3 H^{2}\right)}
$$

Or, equivalently in terms of $\widetilde{H}$ as follows:

$$
\begin{gathered}
r=1-\frac{(1+z)}{\widetilde{H}^{2}} \frac{d \widetilde{H}^{2}}{d z}+\frac{(1+z)^{2}}{2 \widetilde{H}^{2}} \frac{d^{2} \widetilde{H}^{2}}{d z^{2}} \\
s=\frac{-\frac{(1+z)}{\widetilde{H}^{2}} \frac{d \widetilde{H}^{2}}{d z}+\frac{(1+z)^{2}}{2 \widetilde{H}^{2}} \frac{d^{2} \widetilde{H}^{2}}{d z^{2}}}{3\left(\frac{(1+z)}{\widetilde{H}^{2}} \frac{d \widetilde{H}^{2}}{d z}-3\right)}
\end{gathered}
$$

where the explicit form of $r$ is as

$$
\begin{aligned}
& r=1-\left(3 6 ( \frac { 1 } { 1 + z } ) ^ { - 1 + \frac { 3 } { 2 } ( 1 + \omega _ { m } ) } ( J _ { U } ( V ) + J _ { - U } ( V ) C ) H _ { 0 } ^ { 2 } \left(\left(( \frac { 1 } { 1 + z } ) ^ { 1 - \frac { 3 } { 2 } ( 1 + \omega _ { m } ) } \sqrt { - \frac { 1 } { \beta } \beta } \left(\left(\frac{1}{1+z}\right)^{\frac{3}{2}\left(1+\omega_{m}\right)} \alpha \sqrt{-\frac{1}{\beta}} J_{-U}(V) C+\right.\right.\right.\right. \\
& \left.\left.\sqrt{6}\left(\frac{1}{1+z}\right)^{3 \delta / 2}\left(2 J_{-1+U}(V)+\left(J_{-1-U}(V)-J_{1-U}(V)\right) C\right) \sqrt{-\frac{\rho_{m} 0}{\beta}}\right)\left(1+\omega_{m}\right)\right) /\left(24\left(J_{U}(V)+J_{-U}(V) C\right) H_{0}^{2}\right)+ \\
& \left(( \frac { 1 } { 1 + z } ) ^ { - \frac { 3 } { 2 } ( 1 + \omega _ { m } ) } \sqrt { - \frac { 1 } { \beta } } \beta \left(-3 \sqrt{\frac{3}{2}}\left(\frac{1}{1+z}\right)^{1+\frac{3 \delta}{2}} \delta\left(2 J_{-1+U}(V)+\left(J_{-1-U}(V)-J_{1-U}(V)\right) C\right) \sqrt{-\frac{\rho_{m 0}}{\beta}}-\right.\right. \\
& \left.\left.\left.\left.\frac{3}{2}\left(\frac{1}{1+z}\right)^{1+\frac{3}{2}\left(1+\omega_{m}\right)} \alpha \sqrt{-\frac{1}{\beta}} J_{-U}(V) C\left(1+\omega_{m}\right)\right)\right) /\left(36\left(J_{U}(V)+J_{-U}(V) C\right) H_{0}^{2}\right)\right)\right) /\left(\sqrt{-\frac{1}{\beta}} \beta\right. \\
& \left.\left(\left(\frac{1}{1+z}\right)^{\frac{3}{2}\left(1+\omega_{m}\right)} \alpha \sqrt{-\frac{1}{\beta}} J_{-U}(V) C+\sqrt{6}\left(\frac{1}{1+z}\right)^{3 \delta / 2}\left(2 J_{-1+U}(V)+\left(J_{-1-U}(V)-J_{1-U}(V)\right) C\right) \sqrt{-\frac{\rho_{m 0}}{\beta}}\right)\right)+ \\
& \left(1 8 ( \frac { 1 } { 1 + z } ) ^ { - 2 + \frac { 3 } { 2 } ( 1 + \omega _ { m } ) } ( J _ { U } ( V ) + J _ { - U } ( V ) C ) H _ { 0 } ^ { 2 } \left(-\left(( \frac { 1 } { 1 + z } ) ^ { 2 - \frac { 3 } { 2 } ( 1 + \omega _ { m } ) } \sqrt { - \frac { 1 } { \beta } \beta } \left(\left(\frac{1}{1+z}\right)^{\frac{3}{2}\left(1+\omega_{m}\right)} \alpha \sqrt{-\frac{1}{\beta}} J_{-U}(V) C+\right.\right.\right.\right. \\
& \left.\left.\sqrt{6}\left(\frac{1}{1+z}\right)^{3 \delta / 2}\left(2 J_{-1+U}(V)+\left(J_{-1-U}(V)-J_{1-U}(V)\right) C\right) \sqrt{-\frac{\rho_{m 0}}{\beta}}\right)\left(1+\omega_{m}\right)\left(1-\frac{3}{2}\left(1+\omega_{m}\right)\right)\right) /\left(24\left(J_{U}(V)+J_{-U}(V) C\right) H_{0}^{2}\right) \\
& +\left(( \frac { 1 } { 1 + z } ) ^ { 1 - \frac { 3 } { 2 } ( 1 + \omega _ { m } ) } \sqrt { - \frac { 1 } { \beta } } \beta ( 1 + \omega _ { m } ) \left(-3 \sqrt{\frac{3}{2}}\left(\frac{1}{1+z}\right)^{1+\frac{3 \delta}{2}} \delta\left(2 J_{-1+U}(V)+\left(J_{-1-U}(V)-J_{1-U}(V)\right) C\right) \sqrt{-\frac{\rho_{m 0}}{\beta}}-\right.\right. \\
& \left.\left.\frac{3}{2}\left(\frac{1}{1+z}\right)^{1+\frac{3}{2}\left(1+\omega_{m}\right)} \alpha \sqrt{-\frac{1}{\beta}} J_{-U}(V) C\left(1+\omega_{m}\right)\right)\right) /\left(12\left(J_{U}(V)+J_{-U}(V) C\right) H_{0}^{2}\right)+\left(\left(\frac{1}{1+z}\right)^{-\frac{3}{2}\left(1+\omega_{m}\right)} \sqrt{-\frac{1}{\beta}} \beta\right. \\
& \left(3 \sqrt{\frac{3}{2}}\left(\frac{1}{1+z}\right)^{2+\frac{3 \delta}{2}} \delta\left(1+\frac{3 \delta}{2}\right)\left(2 J_{-1+U}(V)+\left(J_{-1-U}(V)-J_{1-U}(V)\right) C\right) \sqrt{-\frac{\rho_{m 0}}{\beta}}+\right. \\
& \left.\left.\left.\left.\frac{3}{2}\left(\frac{1}{1+z}\right)^{2+\frac{3}{2}\left(1+\omega_{m}\right)} \alpha \sqrt{-\frac{1}{\beta}} J_{-U}(V) C\left(1+\omega_{m}\right)\left(1+\frac{3}{2}\left(1+\omega_{m}\right)\right)\right)\right) /\left(36\left(J_{U}(V)+J_{-U}(V) C\right) H_{0}^{2}\right)\right)\right) / \\
& \left(\sqrt{-\frac{1}{\beta} \beta}\left(\left(\frac{1}{1+z}\right)^{\frac{3}{2}\left(1+\omega_{m}\right)} \alpha \sqrt{-\frac{1}{\beta}} J_{-U}(V) C+\sqrt{6}\left(\frac{1}{1+z}\right)^{3 \delta / 2}\left(2 J_{-1+U}(V)+\left(J_{-1-U}(V)-J_{1-U}(V)\right) C\right) \sqrt{-\frac{\rho_{m 0}}{\beta}}\right)\right)
\end{aligned}
$$

\subsection{Cosmographic parameters}

Standard candle is an astronomical ideal object that has a known absolute magnitude. It is extremely important since by measuring the apparent magnitude of the object, the distance can also be determine. It helps to reconstruct the Hubble diagram, i.e, the redshift - distance relation up to high redshift values and for that it is commonly used to parameterized model where it can verify the viability of the data and characterizing parameters. There were some uncertainty as the method is model dependent. Therefore some doubts come to mind when we examine the validity of derived quantities depending on the present day values of the deceleration parameters and the age of the universe. In order to conquer such problem we need a model independent approach and for that the alternative choice is cosmography where the scale factor is expanded in Taylor series with respect to the cosmic time. Such expansions not only show the way to distance-redshift relation, it also supports the assumption of the Robertson-Walker metric. It does not depend on the particular form of the solution of cosmic equations. For that aim, the Cosmographic parameters that are proportional to the coefficients of Taylor series expansion of the scale factor with respect to the cosmic time defined as

$$
q=-\frac{1}{a H^{2}} \frac{d^{2} a}{d t^{2}}=-\frac{1}{H} \frac{d}{d a}(a H)=-1+\frac{(1+z)}{\widetilde{H}^{2}} \frac{d \widetilde{H}^{2}}{d z}
$$




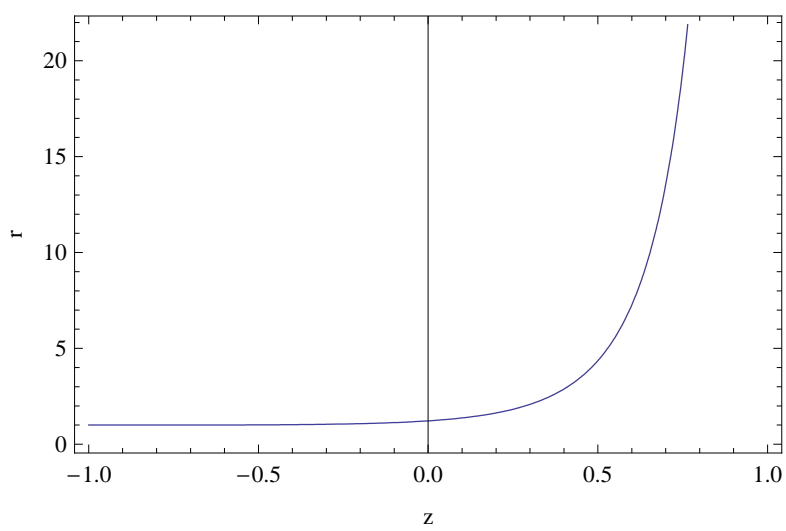

Fig.6

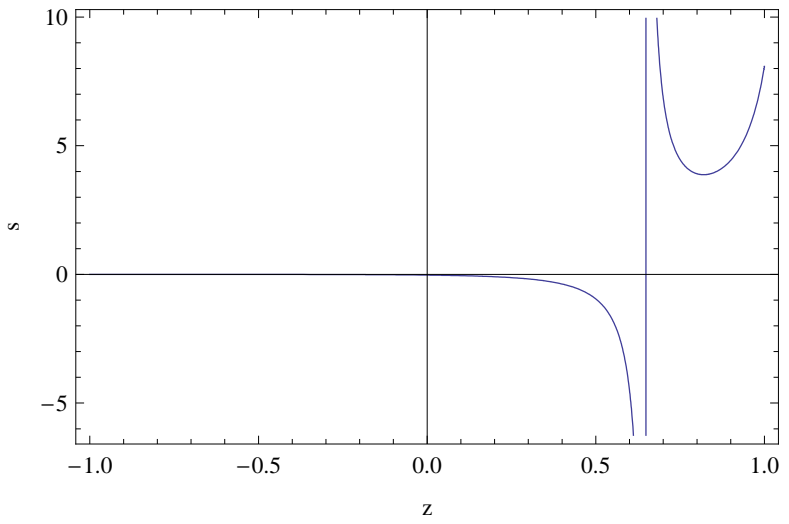

Fig.7

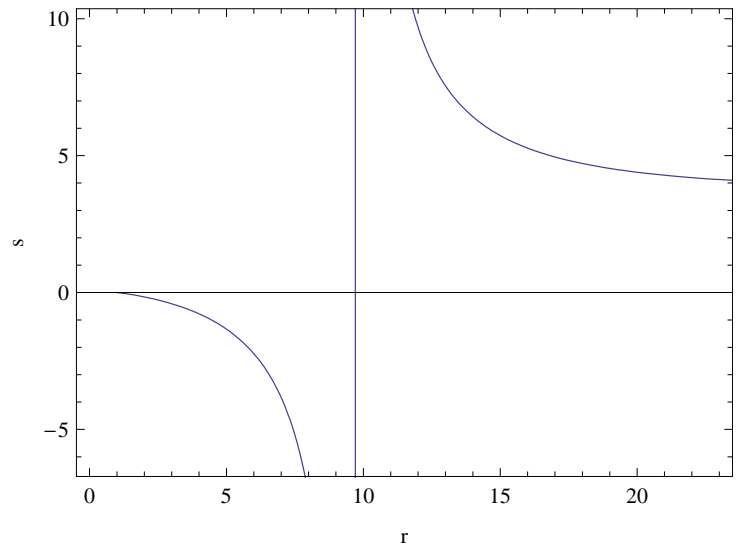

Fig.8

Fig. 6 shows the variation of the state statefinder parameter $r$ with redshift $\mathrm{z}$.

Fig. 7 shows the variation of the state statefinder parameter $\mathrm{s}$ with redshift $\mathrm{z}$.

Fig. 8 shows the variation of the state statefinder parameter $\mathrm{s}$ against $\mathrm{r}$. 


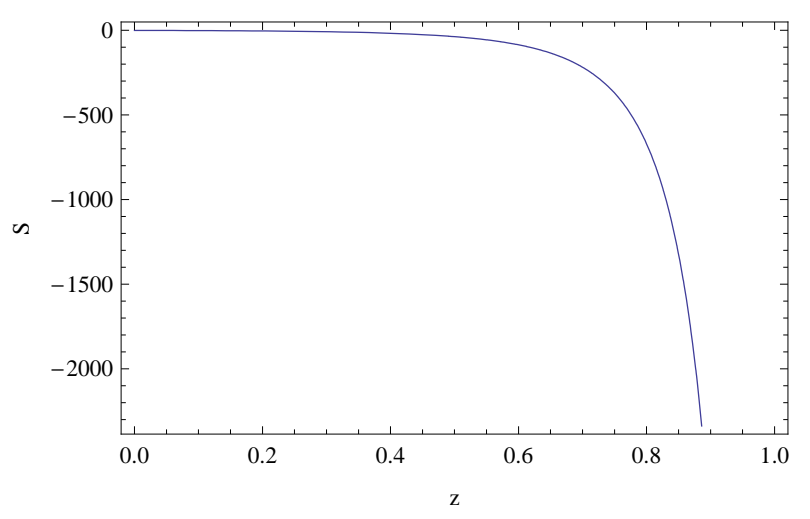

Fig.9

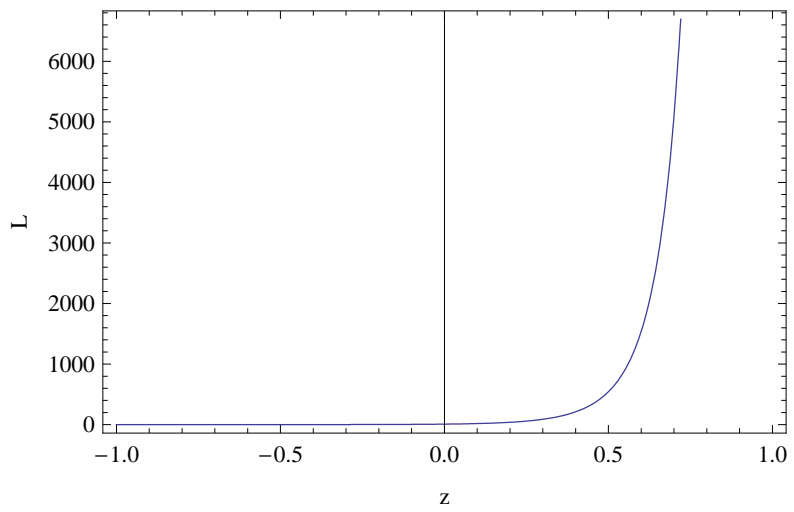

Fig.10

Fig. 9 shows the variation of the Snap parameter $\mathrm{S}$ with redshift $\mathrm{z}$.

Fig. 10 shows the variation of the Lerk parameter $\mathrm{L}$ with redshift $\mathrm{z}$.

$$
\begin{gathered}
J=r=\frac{1}{a H^{3}} \frac{d^{3} a}{d t^{3}}=-\frac{1}{H^{2}} \frac{d}{d a}\left(a H^{2} q\right)=1-\frac{(1+z)}{\widetilde{H}^{2}} \frac{d \widetilde{H}^{2}}{d z}+\frac{(1+z)^{2}}{2 \widetilde{H}^{2}} \frac{d^{2} \widetilde{H}^{2}}{d z^{2}} \\
S=\frac{1}{a H^{4}} \frac{d^{4} a}{d t^{4}}=\frac{1}{H^{3}} \frac{d}{d a}\left(a H^{3} J\right)=-\frac{(1+z)^{2}}{\widetilde{H}^{3}} \frac{d}{d z}\left(\frac{\widetilde{H}^{3}}{1+z} J\right) \\
L=\frac{1}{a H^{5}} \frac{d^{5} a}{d t^{5}}=\frac{1}{H^{4}} \frac{d}{d a}\left(a H^{4} S\right)=-\frac{(1+z)^{2}}{\widetilde{H}^{4}} \frac{d}{d z}\left(\frac{\widetilde{H}^{4}}{1+z} S\right)
\end{gathered}
$$

which are usually referred to as the deceleration $(q)$, jerk $(J)$, snap $(S)$ and lerk $(L)$ parameters, respectively. The Cosmographic Parameters's present day values (which are denote with a subscript 0) may be used to characterize the evolutionary status of the universe. For instance, $q_{0}<0$ denotes an accelerated expansion, while $J_{0}$ allows to discriminate among different accelerating models.

\subsection{Om Parameter}

Recently, Sahni et al [50, 51] proposed a new cosmological parameter named Om which was introduced to differentiate $\Lambda \mathrm{CDM}$ from other dark energy models $\mathrm{Om}$ diagnostic has been discussed together with statefinder for generalized Chaplygin gas model from cosmic observations in [52, 53. Generally, it is a combination of Hubble parameter and the cosmological redshift and provides a null test of dark energy. For $\Lambda$ CDM model, $O m=\Omega_{m 0}$ is a constant, independent of redshift $z$. Also it helps to distinguish the present matter density constant $\Omega_{m 0}$ in different models more effectively. The main utility for $\mathrm{Om}$ diagnostic is that the quantity of $\mathrm{Om}$ can distinguish dark energy models with less dependence on matter density $\Omega_{m 0}$ relative to the EoS of dark energy. Our starting point for $\mathrm{Om}$ diagnostics in the Hubble parameter and it is defined as:

$$
O m(z)=\frac{\widetilde{H}^{2}(z)-1}{(1+z)^{3}-1}
$$

Using the above result we have

$$
O m(z)=\frac{\frac{\sqrt{-\frac{\beta}{a^{3\left(1+\omega_{m}\right)}}}\left[\alpha \sqrt{-\frac{a^{3\left(1+\omega_{m}\right)}}{\beta}} J_{-U}(V) C+\sqrt{-\frac{6 a^{3 \delta} \rho_{m 0}}{\beta}}\left(2 J_{-1+U}(V)+\left(J_{-1-U}(V)-J_{1-U}(V)\right) C\right)\right]}{36 H_{0}^{2}\left(J_{U}(V)+J_{-U}(V) C\right)}-1}{(1+z)^{3}-1}
$$

\section{Stability Analysis}

In this section, we have examined the stability of this model. In this connection, the most important quantity is the squared speed of sound which is denoted as $v_{s}^{2}$ and defined as a ratio of the effective pressure and energy densities i.e., $v_{s}^{2}=\frac{\dot{p}}{\dot{\rho}}$. The sign of $v_{s}^{2}$ plays a vital role for stability analysis of a background evolution of cosmic models. It is well known that the model is stable if $v_{s}^{2}>0$ and if $v_{s}^{2}<0$ implies that the model is classically unstable [54. In 2008, Kim et al. 55] found that $v_{s}^{2}$ for agegraphic DE is always negative which leads to classically instability of that model. Recently many researchers are using this methods to analyzed the stability of that models on which they worked and of which some authors [56, 57, 58, 59] have reached to a conclusion that HDE, ADE, NADE, Chaplygin gas, holographic Chaplygin, 


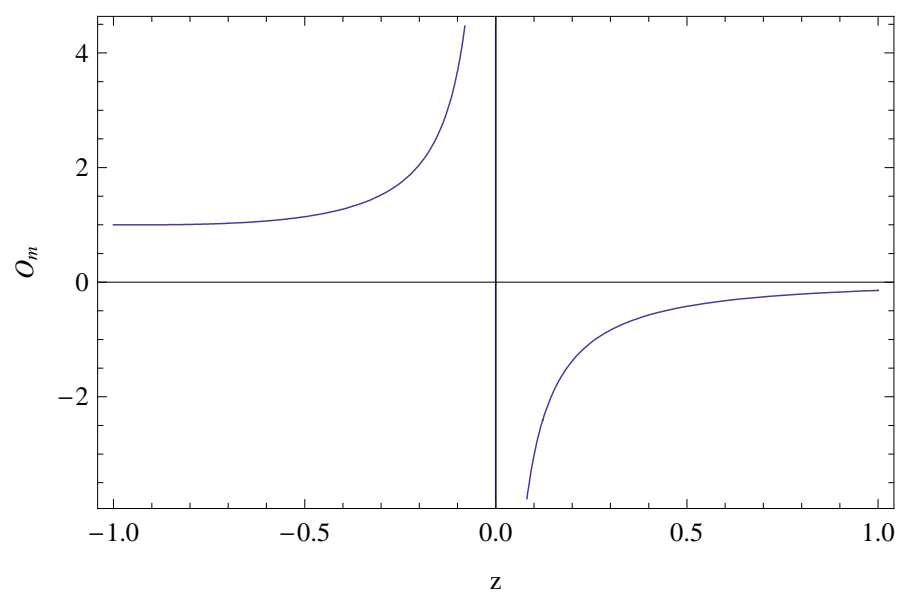

Fig.11

Fig.11 gives the plot of $O m$-diagnostic against redshift parameter $z$

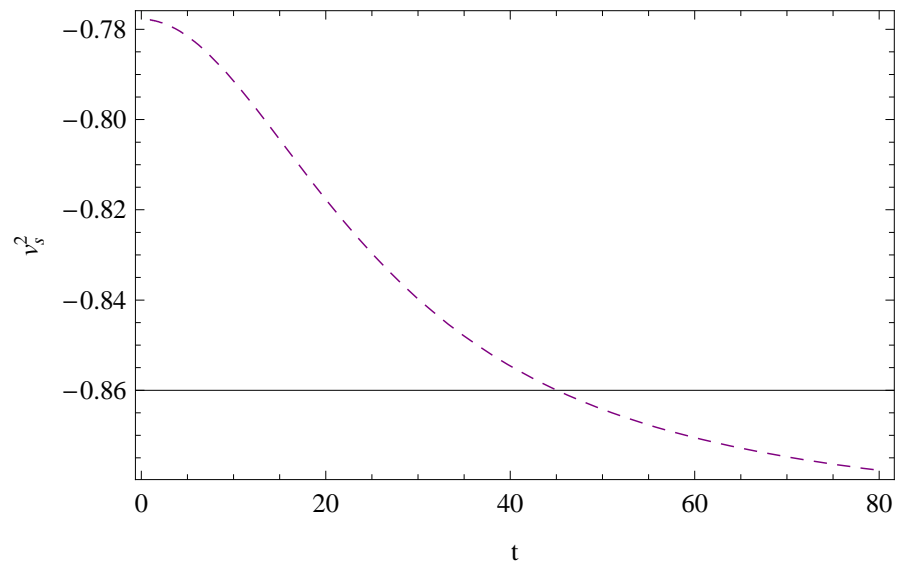

Fig.12

Fig 12 shows the variation of the squared speed of sound $v_{s}^{2}$ against the cosmic time $t$.

holographic $f(T)$, holographic $f(G)$, new agegraphic $f(T)$, new agegraphic $f(G)$ models are classically unstable because squared speed of sound is negative i.e., $v_{s}^{2}<0$ throughout the evolution of the universe. Here we consider

$$
v_{s}^{2}=\frac{\dot{p}_{M G}}{\dot{\rho}_{M G}}
$$

and plot $v_{s}^{2}$ versus $t$ by taking the power-law scale factor in this model. Fig. 12 shows the variation of $v_{s}^{2}$ with $t$ for the above mentioned cases. In this model we observed that the square speed of sound remains negative for the present and future epoch, which implies that our predicted model for the above mentioned scenarios with power-law form of scale factor is classically unstable.

\section{Discussions}

In this work we have studied the behaviour of some cosmological parameters like Deceleration parameter, EoS parameter, Om parameter, State-finder parameters and Cosmographic parameters in an universe described by interacting new holographic dark energy model in $f(T)$ gravity. We have considered a suitable interaction between holographic dark energy and dark matter to account for the coincidence scenario. We have derived the expressions for the above mentioned cosmological parameters and plotted them against the cosmological redshift parameter $z$. 
In fig.1, we have plotted the deceleration parameter against the redshift. From the plot we see that at the current epoch $(z=0)$, the value of $q$ is around -0.8 , which shows that the universe is going through an accelerated expansion. From the curve it is also evident that the universe is evolving from an early decelerating phase to a late accelerating phase. In fig.2, the EoS parameter is plotted against the redshift parameter. It is seen that currently the value of $\omega$ is around -0.9 , which lies in the quintessence range $(-1<\omega<-1 / 3)$. Moreover it is seen that near the $\omega=-1$ axis the curve assumes an asymptotic behaviour, which shows that the universe never enters the phantom regime, although the trajectory is infinitesimally close to the phantom divide. In the figs. 3,4 and 5 , the trajectories in the $\omega$ - $\omega^{\prime}$ plane are obtained for different values of interactions. It is seen that for no or smaller interaction models we get freezing regions which are best suited for the current accelerating scenario. But for high interactions we get thawing models. In the figs. 6 and 7, the statefinder parameters $r$ and $s$ are respectively plotted against the redshift parameter. From the trajectories it is seen that in the present time $r=1, s=0$, which corresponds to the standard $\Lambda$ CDM cosmological model of accelerating universe. In fig. 8, trajectories in the $r-s$ plane are obtained for the given model. This figure also confirms the fact that when $r=1$, we have $s=0$, which is quite characteristic for an accelerating model of the universe. In figs. 9 and 10, the Snap and the Lerk parameters are respectively plotted against the redshift parameter. From fig.9, we see that after passing through a series of negative values in the early universe, it assumes constancy around the zero level in the present universe. Almost similar is the case for the Lerk parameter in fig 10, the only difference being its values lying in the positive region in the early universe. In fig. 11, we have obtained the trajectories for the $\mathrm{Om}$-diagnostic. The trajectories obtained are unique for the given model and can be used to differentiate the model from others. Finally in fig. 12, the squared speed of sound $v_{s}^{2}$ is plotted against cosmic time $t$. From the figure it is seen that the trajectory for the present model remains at the negative level thus exhibiting the unstable nature of the model. Nevertheless the study reveals that the model is perfectly consistent with the notion of the accelerating universe, at least for low interaction cases, and the derived values of all the parameters comply to this fact.

\section{Acknowledgement:}

The authors are thankful to IUCAA, Pune, India for warm hospitality where a part of the work was carried out.

\section{References}

[1] Gold, B. et al., 2009, Astrophys. J. Suppl. 180, 265.

[2] Nolta, M. R. et al., 2009, Astrophys. J. Suppl. 180, 296.

[3] Bachall, N. A., et al., 1999, Science 284,1481.

[4] Perlmutter, S. J., et al., 1999, ApJ. 517,565.

[5] Abazajian, K. et al., 2004, Astron. J. 128, 502.

[6] Abazajian et al., 2005, Astron. J. 129, 1755.

[7] Perlmutter, S. J. et al., 1998, Nature 391,

[8] Riess, A. G. et al.[Supernova Search Team Collaboration], 1998, Astron. J. 116,1009.

[9] Riess, A. G. et al., 2004, Astrophys. J. 607,665.

[10] Bennet, C. et al, 2000, Phys. Rev. Lett. 85,2236.

[11] D. N. Spergel et al., 2007, Astrophys. J. Suppl. Ser. 170,377.

[12] Sahni, V. and Starobinskyet A. A., 2006, Int. J. Mod. Phys. D15,2105.

[13] Sahni, V. and Starobinskyet A. A.,2000, Int. J. Mod. Phys. D 9, 373.

[14] Seikel, M. et al., 2012, JCAP 06, 036.

[15] Clarkson, C. et al., 2010, Phys. Rev. Lett. 104, 211301.

[16] Liu, X. M. et al., 2011, Astrophys. Space Sci. 334,203.

[17] Vollick, D.N., 2007, Phys. Rev. D 76, 124001.

[18] Briscese, F., et al., 2007, Phys. Lett. B 646, 105.

[19] Carroll, S.M., et al., 2004, Phys. Rev. D 70, 043528.

[20] Abdalla, M.C.B., et al., 2005, Class. Quantum Grav. 22, L35.

[21] E.V. Linder, 2010, Phys. Rev. D 81, 127301.

[22] Nojiri, S., et al., 2003, Phys. Rev. D 68, 123512.

[23] Nojiri, S., et al., 2007, Int. J. Geom. Methods Mod. Phys. 4, 115. 
[24] Nojiri, S., et al., 2008, Phys. Lett. B 659, 821.

[25] Li, B. and Barrow, J.D., 2007, Phys. Rev. D 75, 084010.

[26] Nojiri, S., Odintsov, S.D., 2006, Phys. Rev. D 74, 086005.

[27] Li, B. et al., 2011, Phys. Rev. D 83, 104017.

[28] Myrzakulov, R., 2011, Eur. Phys. J. C 71, 1752.

[29] Wu P. et al., 2011, Eur. Phys. J. C 71, 1552.

[30] Rastkar, A.R. et al., 2012, Astrophys. Space Sci. 337, 487.

[31] Nojiri, S., Odintsov, S.D., 2005, Phys. Lett. B 631, 1.

[32] Harko, T. et al., 2011, Phys. Rev. D 84, 024020.

[33] Jamil, M. et al., 2012, Eur. Phys. J. C 72, 1999.

[34] Bamba, K. et al., 2010, Eur. Phys. J. C 67, 295.

[35] Myrzakulov, R. et al., 2013, Gen Relativ Gravit 45, 675.

[36] Setare, M.R. et al., 2008, Phys. Lett. B 670, 1.

[37] Liu, X.L., et al., 2009, Commun. Theor. Phys. 52, 761.

[38] Setare, M.R., 2010, Astrophys. Space Sci. 326, 27.

[39] Jamil, M., et al., 2010, JCAP 07, 028.

[40] Setare, M. R., et al., 2010 EPL 92, 49003.

[41] Jawad, A. et al., 2013 Astrophys. Space Sci. 344, 489.

[42] Caldwell, R.R., Linder, E.V., 2005, Phys. Rev. Lett. 95, 141301.

[43] Scherrer, R.J., 2006, Phys. Rev. D 73, 043502.

[44] Sharif, M., Jawad, A., 2013a, Eur. Phys. J. C 73, 2382.

[45] Sharif, M., Jawad, A., 2013b, Eur. Phys. J. C 73, 2600.

[46] Sharif, M., Jawad, A., 2014, Astrophys. Space Sci. 351, 321.

[47] Guo, Z.K., et al., 2006, Phys. Rev. D 74, 127304.

[48] Chiba, T., 2006, Phys. Rev. D 73, 063501.

[49] Malekjani, M., Khodam-Mohammadi, A., 2012, Int. J. Theor. Phys. 51, 3141.

[50] Sahni, V., Saini, T. D., Starobinsky A. A. and Alam, U., 2003, JETP Lett., 77, 201.

[51] Sahni, V., et al., 2008, Phys. Rev. D, 78,103502.

[52] Lu, J., et al., 2009, Int. J. Mod. Phys. D, 18, 1741.

[53] Tong M. L. and Zhang, Y., 2009, Phys. Rev. D, 80, 023503.

[54] Myung, Y. S., 2007, Phys. Lett. B, 652, 223.

[55] Kim, K. Y.,Lee, H. W. and Myung, Y. S., 2008, Phys. Lett. B 660, 118.

[56] Jawad, A., Chattopadhyay, S. and Pasqua, A., 2013, Eur. Phys. J. Plus, 128, 88.

[57] Ebrahimi, E. and Sheykhi, A., 2011, Int. J. Mod. Phys. D 20, 2369.

[58] Sharif, M. and Jawad, A., 2012, Eur. Phys. J. C 72, 2097.

[59] Setare, M. R., 2007, Phys. Lett. B 654, 1. 